\title{
Flu DRiPs in MHC Class I Immunosurveillance
}

\author{
Jiajie Wei ${ }^{1} \cdot$ Jonathan W. Yewdell ${ }^{1}$ (I)
}

Received: 28 August 2018/Accepted: 30 September 2018/Published online: 19 November 2018

(C) The Author(s) 2018

\begin{abstract}
Since the publication of the DRiP (defective ribosomal product) hypothesis in 1996, numerous studies have addressed the contribution of DRiPs to generating viral antigenic peptides for $\mathrm{CD}^{+} \mathrm{T}$ cell immunosurveillance. Here, we review studies characterizing the generation of antigenic peptides from influenza A virus encoded DRiPs, discuss the many remaining mysteries regarding the nature of their co-translational generation, and speculate on where the future might lead.
\end{abstract}

Keywords Influenza A virus (IAV) - MHC-restricted cytotoxicity $\cdot$ Immune responses $\cdot$ MHC class I $\cdot$ DRiP

\section{Introduction}

Influenza A virus (IAV), a negative-sense, single-stranded, segmented RNA virus, causes significant worldwide morbidity, mortality and economic burden due to its evasion of adaptive immunity, despite repeated infection and vaccination. $\mathrm{CD}^{+} \mathrm{T}$ cell immunosurveillance of $\mathrm{MHC}$ class I-peptide complexes, a crucial part of the adaptive immune system, recognizes peptides encoded by each of the eight IAV gene segments, limits viral replication and reduces morbidity and mortality in hosts whose antibody responses fail to prevent infection (McMichael et al. 1983; Kim et al. 2011). Vaccines that induce and boost $\mathrm{CD} 8^{+} \mathrm{T}$ cells are promising candidates for improving the duration of effective immunity to IAV (Souquette and Thomas 2018), since many of the immunogenic IAV peptides are highly conserved among human IAV isolates.

By binding and presenting oligopeptides at the cell surface, MHC class I molecules provide a window into the translational status of cells. This enables $\mathrm{CD}^{+} \mathrm{T}$ cell recognition of viruses and other intracellular pathogens, cancers, transplants and autoimmune targets. MHC class I molecules also function in other biological processes, including neutral killer cell activation, mate selection, and

Jonathan W. Yewdell

jyewdell@nih.gov

1 Cellular Biology Section, Laboratory of Viral Diseases, National Institute of Allergy and Infectious Diseases, National Institutes of Health, Bethesda, MD 20892, USA neuronal development (McAllister 2014; Apanius et al. 2017).

Viruses have played a central role in understanding the MHC class I biology, starting from the discovery of MHC restriction, which provided the first clear evolutionary justification for a system discovered based on its prominent role in graft rejection (Zinkernagel and Doherty 1974). $\mathrm{CD}^{+} \mathrm{T}$ cells play an important role in the clearance of many viruses. The sheer number and variety of viral proteins known to interfere with various steps of the class I pathway underscores the importance of antigen presentation in viral immunity and viral evolution (Schuren et al. 2016).

MHC class I antigenic peptides encoded by host or viral genes have two potential sources: "retirees" (Yewdell 2001, 2003) and DRiPs (defective ribosomal products) (Yewdell et al. 1996). Retirees are proteins that reach stable structures and degrade with normal turnover kinetics, i.e. a median half-life of $46 \mathrm{~h}$ across the entire proteome (Schwanhäusser et al. 2011). DRiPs, a substantial subset of nascent gene products that degrade more rapidly than their corresponding native retiree pools (Wheatley et al. 1980; Schubert et al. 2000; Wang et al. 2013), were originally proposed to explain the rapidity of $\mathrm{CD}^{+} \mathrm{T}$ cell recognition of virus infected cells. Viral peptide ligands can be generated well within an hour after viruses are added to cells (Esquivel et al. 1992; Croft et al. 2013; Zanker et al. 2013).

DRiPs were originally conceived as misfolded or prematurely terminated proteins arising as inevitable errors in protein biogenesis and errors deliberated enhanced by 
innate cellular responses to infection (Yewdell et al. 1996). The DRiP hypothesis has evolved over the years (Yewdell 2003, 2011; Yewdell and Nicchitta 2006; Anton and Yewdell 2014) to encompass all possible errors that can occur in converting genetic information into mature proteins, including stoichiometric excess subunits of multiprotein complexes (Bourdetsky et al. 2014), and products of non-canonical translation and mistranslation. Importantly, numerous studies support that immunological relevant viral peptides predominately originate from DRiPs, including products from downstream initiation on AUG codons (Berglund et al. 2007), frame shifting (Fetten et al. 1991; Bullock and Eisenlohr 1996; Elliott et al. 1996; Zook et al. 2006), initiation on non-AUG start codons (Yang et al. 2016), stop codon read through, and translation of viral RNA in the nucleus (Dolan et al. 2010a).

\section{Flu DRiPs}

The first studies demonstrating that peptides can efficiently arise from rapidly degraded defective proteins came from Alain Townsend's pioneering research showing that $\mathrm{CD} 8^{+}$ $\mathrm{T}$ cells lyse cells expressing rapidly degraded versions of truncated influenza proteins expressed from transgenes (Townsend et al. 1985, 1986). These findings were extended by Fetten et al. (1991) who found that presentation of a NP peptide to $\mathrm{CD} 8^{+} \mathrm{T}$ cells continued apace after introduction of an upstream frameshift that completely abrogated detection of full length NP encoded by a retrovirus transduced gene. These findings were extended to mutated IAV proteins expressed by recombinant vaccinia viruses (Bullock and Eisenlohr 1996; Elliott et al. 1996), which became a workhorse for mechanistic antigen processing studies due to the relative ease of generating recombinant viruses that could be used to infect many cells lines in vitro as well as animals in vivo.

With time, it became possible to use IAV itself to study the effects of genetic alterations on class I peptide generation (Webby et al. 2003). This was an essential step to understanding IAV peptide generation, since the rules of generating peptides are likely to vary depending on the exact nature of how the protein is synthesized, which varies considerably among different virus familes. For example, we found that the same protein encoded by a vaccinia virus generated mRNA $v s$. a host cell transgene encoded-mRNA generates peptide with different efficiencies per retiree molecule degraded (Dolan et al. 2012). Still, the small coding capacity of IAV sets a fairly tight limit on adding genetic information and the need for the virus to replicate limits genetic manipulation of essential genes.

The engineering of the model $\mathrm{H} 2-\mathrm{K}^{\mathrm{b}}$ binding 8-mer SIINFEKL peptide into IAV (Jenkins et al. 2006), in combination with $\mathrm{K}^{\mathrm{b}}$-SIIINFEKL detection reagents $\mathrm{B} 3 \mathrm{Z}$ hybridoma cells (Karttunen et al. 1992), OT-I TCR transgenic mice (Clarke et al. 2000), and the 25-D1.16 monoclonal antibody (Porgador et al. 1997), provides an invaluable system for sensitively and quantitatively studying peptide generation in vitro and in mice. To study natural IAV DRiPs, Dolan et al. genetically inserted SIINFEKL into the stalk of IAV neuraminidase (NASIINFEKL) and monitored $\mathrm{K}^{\mathrm{b}}$-SIINFEKL presentation on cell surface after IAV infection (Dolan et al. 2010b). As far as known, the only function of the NA stalk is to set the height of NA globular domain, and stalk length varies widely among various circulating NA genes in the animal reservoir. Indeed, SIINFEKL insertion did not change NA translation, folding, degradation, transport, or surface expression. Kinetic analysis using 25-D1.16 for flow cytometric determination of $\mathrm{K}^{\mathrm{b}}$-SIINFEKL cell surface generation revealed that $\mathrm{K}^{\mathrm{b}}$-SIINFEKL is generated in lockstep with initiation and abrogation of NA synthesis in both $\mathrm{L}-\mathrm{K}^{\mathrm{b}}$ fibroblast cells and DC2.4 dendritic/monocyte cells, supporting the importance of natural viral DRiPs during infection.

Dolan et al. (2010a) reported an intriguing disconnect between standard NA-SIINFEKL translation and $\mathrm{K}^{\mathrm{b}}$ SIINFEKL generation. Unlike most RNA viruses, IAV (and other myxoviruses) express their mRNA in the nucleus, where the mRNAs steal caps from cellular mRNA to enable their export from the nucleus and translation. RNA polymerase (RNAP) II inhibitors, 5,6-dichloro-1-b-D-ribofuranosylbenzimidazole (DRB) and actinomycin D, prevent nuclear of export some IAV mRNAs (Amorim et al. 2007), including NA, as Dolan et al. reported. Remarkably, a 33-fold reduction of NA expression in DRB treated cells was accompanied by only a 5-fold decrease in $\mathrm{K}^{\mathrm{b}}$-SIINFEKL cell surface expression. This is consistent with the idea that translation of NASIINFEKL RNA in the nucleus accounts for a fraction of antigenic peptide generation from NA.

Nuclear translation is a highly contentious topic (Dahlberg et al. 2003; Iborra et al. 2004; David et al. 2012; Reid and Nicchitta 2012; Al-Jubran et al. 2013), and its resurrection stirred the hornet's nest (Dahlberg and Lund 2012). Its possible contribution to immunosurveillance was further supported by studies showing that OT-I $\mathrm{T}$ cells equally recognize cells expressing SIINFEKL from introns $v s$. exons in transfected genes (Apcher et al. 2013). $\mathrm{K}^{\mathrm{b}}$-SIINFEKL generation from both exons and intronic SIINFEKL was unaffected by blocking mRNA export from the nucleus, suggesting nuclear translation of prespliced RNA as a surprising source for peptide generation.

Over several decades the Shastri lab pioneered studies demonstrating the usage of non-canonical translation start sites for MHC class I peptide immunosurveillance. With 
the Pan lab, they discovered that antigenic peptides can be initiated from CUG with an elongator leucine-tRNA rather than the canonical AUG start codon with Met-tRNA (Starck et al. 2012). This translation is based on initiation factor eIF2 rather than canonical eIF2A initiation (Starck et al. 2016). Yang et al. (2016) extended these findings to IAV immunosurveillance by studying the generation of $\mathrm{K}^{\mathrm{b}}$ SIINFEKL complexes from the IAV M2 gene with SIINFEKL at its C-terminus. IAV exploits its nuclear transcription to create a number of spliced mRNAs. Gene segment seven encodes two proteins: M1 is translated from unspliced mRNA while all but the 9-N-terminal residues in M2 encoded by the M1 +1 reading frame from a spliced mRNA. Yang found that while the mRNA splicing inhibitor spliceostatin A nearly completely inhibited M2 mRNA generation and protein synthesis, $\mathrm{K}^{\mathrm{b}}$-SIINFEKL complexes were still robustly generated. The use of CUG codons in unspliced M1 mRNA as initiation codons was supported by three lines of evidence. First, $\mathrm{K}^{\mathrm{b}}$-SIINFEKL was generated in vitro and in vivo from mRNA synthesized in the cytoplasm by vaccinia virus, whose mRNAs are not known to be subject to splicing, which is thought to only occur in the nucleus. Second, drugs that block AUG initiation did not reduce the level of $K^{\mathrm{b}}$-SIINFEKL complexes. Third, and most conclusively, synonymous mutation of CUG codons severely reduced $\mathrm{K}^{\mathrm{b}}$-SIINFEKL generation. Thus, Yang et al. defined an IAV DRiP generated by cytoplasmic noncanonical translation, and demonstrates the participation of CUG-codon-based translation initiation in pathogen immunosurveillance.

Even more remarkably, although the negative stranded genomic RNA of IAV is nearly "non-coding" by definition, Hickman et al. (2018) found that $\mathrm{K}^{\mathrm{b}}$-SIINFEKL complexes can be generated from two different negative strand gene segments. A long open reading frame that is highly conserved among human and animal IAVs is present in the genomic strand of segment eight, encoding a potential $\sim 167$ residue protein termed NEG8 (Zhirnov et al. 2007; Clifford et al. 2009). Despite a lack of biochemical evidence of NEG8 protein expression, $\mathrm{K}^{\mathrm{b}}$-SIINFEKL complexes are generated when SIINFEKL is appended to the predicated COOH-terminus of NEG8, since infection with the recombinant virus activates OT-I T cells in vitro and in vivo. SIINFEKL embedded in the negative strand of the NA-stalk coding sequence also activates OT-I T cells in vivo, albeit weakly. These findings demonstrate translation of the IAV negative strand can also contribute to DRiPs generation and anti-viral immunosurveillance. The fascinating questions of where in the cell (nucleus vs. cytoplasm) and how the IAV negative stand is translated remain to be investigated.

\section{IAV DRiPomics}

A central aspect of the DRiPs theory holds that DRiPs are an inevitable result of protein translation. Although the biochemical nature of DRiPs generated from viral proteins remains a work very much in progress, mass spectrometrybased studies that correlate viral protein synthesis with peptide generation provide definitive evidence for DRiPs as a major source of viral peptides. In a groundbreaking study, Croft et al. (2013) used a novel approach, liquid chromatography coupled with a targeted variation of mass spectrometry termed multiple reaction monitoring, to quantify eight vaccinia virus immunogenic peptides and their source proteins at multiple time points after infecting cultured cells. This revealed a tight correlation between the onset of protein expression and peptide presentation, providing the strongest biochemical evidence to date for the central contribution of DRiPs to presentation of viral peptides during acute cell infection.

Wu extended this approach to IAV (2017), identifying 22 IAV-derived class I bound peptides including eight previously unknown peptides. Peptides bound by surface MHC-I on IAV infected DC2.4 cells were then quantified across a time course of infection with the abundance of viral source proteins measured simultaneously. Importantly, nearly all of the viral peptides were detected prior to or simultaneously with their source proteins, clearly demonstrating the general relevance of DRiPs to IAV immunosurveillance.

\section{Future Directions}

The next step in defining the contribution of DRiPs to viral immunosurveillance is to use ribosome profiling (RiboSeq) to identify all possible translation events. In RiboSeq, RNase resistant ribosome protected fragments are deep sequenced in conjunction with the use of translation inhibitors to enable identification of initiation, stalling, and termination events (Ingolia et al. 2011, 2014). The frequency of translation is inferred by read numbers of translated sequences. Despite the power and progressive optimization of RiboSeq (McGlincy and Ingolia 2017), the method is challenging both technically and informatically.

While information from RiboSeq will be essential in unraveling the mysteries in DRiP generation and will likely identify additional immunogenic IAV peptides, other approaches will also be important. The efficiency of generating class I binding peptides from DRiPs can differ several fold from highly similar virus-encoded substrates, pointing to important differences in exactly how proteins are delivered to proteasomes for degradation (Princiotta 
et al. 2003). Further, intracellular peptide competition studies showed that while preprocessed peptides competed for class I presentation as expected from the law of mass action, they are unable to inhibit presentation by peptides liberated from DRiPs (Lev et al. 2010), consistent with the idea of compartmentalized translation, degradation, and delivery of peptides to the TAP. Further, the burgeoning heterogeneity in ribosome structures (Slavov et al. 2015; Shi et al. 2017; Genuth and Barna 2018), ribosomes themselves are likely to have modifications that modulate their ability to generate antigenic peptides (Yewdell and Nicchitta 2006; Wei and Yewdell 2018).

Adding to the complexity and interest, a recent report concludes that $30 \%$ of class I peptides are generated by peptide splicing (Liepe et al. 2016), presumably via the proteasome (Hanada et al. 2004; Vigneron et al. 2004; Warren et al. 2006). As proteasome-mediated splicing is an uncommon, though potentially efficient event using purified proteasomes (Berkers et al. 2015), this points to the possibility of special treatment of spliced peptides by the class I pathway. It will certainly be of great interest to explore the contribution of peptide splicing to viral immunosurveillance.

Finally, it is highly likely that tissue and cell type specific viral epitopes exist and are exploited by the immune system. In vivo studies that combine RiboSeq and mass spec of MHC-I peptides, although technically challenging given the large amount of starting material needed, are absolutely needed to validate and extend findings with cultured cells.

\section{Working Together to Improve Global Health}

This review was written to commemorate the highly successful visit of NIH virologists in March 2018 to Wuhan and Beijing to meet Chinese virologists and share their expertise and knowledge. Among all pathogens, viruses pose the greatest risk to humanity due to their ubiquity and ability to rapidly mutate. Viruses do not respect political boundaries between countries. With the ever expanding human population, increased travel of individuals between once isolated locales, and man-made rapid changes in climate that alter local ecologies, the risk for catastrophic viral pandemics constantly increases. Increased communication between virologists, including organized mechanisms for sharing data, reagents and people, will be essential to minimize the impact of viruses on human health.

On a personal note, one of the joys as a senior scientist (JWY), is to work with talented and enthusiastic young scientists from around the globe. In the course of my career at NIH, I have had the great pleasure of working with seven post-docs from China, all of whom made important discoveries in my laboratory. I thank the people of China for raising and training such outstanding people, and to urge that the scientific ties between our countries, and all countries, continue to increase.

The fate of the world depends on it!

Acknowledgements The authors are supported by the Division of Intramural Research, NIAID, NIH.

\section{Compliance with Ethical Standards}

Conflict of interest The authors declare that they have no conflict of interest.

Animal and Human Rights Statement The authors declare that they have no conflict of interest. This article does not contain any studies with human or animal subjects performed by any of the authors.

Open Access This article is distributed under the terms of the Creative Commons Attribution 4.0 International License (http://creative commons.org/licenses/by/4.0/), which permits unrestricted use, distribution, and reproduction in any medium, provided you give appropriate credit to the original author(s) and the source, provide a link to the Creative Commons license, and indicate if changes were made.

\section{References}

Al-Jubran K, Wen J, Abdullahi A, Roy Chaudhury S, Li M, Ramanathan P, Matina A, De S, Piechocki K, Rugjee KN, Brogna S (2013) Visualization of the joining of ribosomal subunits reveals the presence of $80 \mathrm{~S}$ ribosomes in the nucleus. RNA 19:1669-1683

Amorim MJ, Read EK, Dalton RM, Medcalf L, Digard P (2007) Nuclear export of influenza A virus mRNAs requires ongoing RNA polymerase II activity. Traffic 8:1-11

Anton LC, Yewdell JW (2014) Translating DRiPs: MHC class I immunosurveillance of pathogens and tumors. J Leukoc Biol 95:551-562

Apanius V, Penn D, Slev PR, Ruff LR, Potts WK (2017) The nature of selection on the major histocompatibility complex. Crit Rev Immunol 37:75-120

Apcher S, Millot G, Daskalogianni C, Scherl A, Manoury B, Fahraeus $R$ (2013) Translation of pre-spliced RNAs in the nuclear compartment generates peptides for the MHC class I pathway. Proc Natl Acad Sci USA 110:17951-17956

Berglund P, Finzi D, Bennink JR, Yewdell JW (2007) Viral alteration of cellular translational machinery increases defective ribosomal products. J Virol 81:7220-7229

Berkers CR, de Jong A, Schuurman KG, Linnemann C, Meiring HD, Janssen L, Neefjes JJ, Schumacher TN, Rodenko B, Ovaa H (2015) Definition of proteasomal peptide splicing rules for highefficiency spliced peptide presentation by MHC class I molecules. J Immunol 195:4085-4095

Bourdetsky D, Schmelzer CE, Admon A (2014) The nature and extent of contributions by defective ribosome products to the HLA peptidome. Proc Natl Acad Sci USA 111:E1591-1599

Bullock TN, Eisenlohr LC (1996) Ribosomal scanning past the primary initiation codon as a mechanism for expression of CTL epitopes encoded in alternative reading frames. J Exp Med 184:1319-1329 
Clarke SR, Barnden M, Kurts C, Carbone FR, Miller JF, Heath WR (2000) Characterization of the ovalbumin-specific TCR transgenic line OT-I: MHC elements for positive and negative selection. Immunol Cell Biol 78:110-117

Clifford M, Twigg J, Upton C (2009) Evidence for a novel gene associated with human influenza A viruses. Virol J 6:198

Croft NP, Smith SA, Wong YC, Tan CT, Dudek NL, Flesch IE, Lin LC, Tscharke DC, Purcell AW (2013) Kinetics of antigen expression and epitope presentation during virus infection. PLoS Pathog 9:e1003129

Dahlberg J, Lund E (2012) Nuclear translation or nuclear peptidyl transferase? Nucleus 3:320-321

Dahlberg JE, Lund E, Goodwin EB (2003) Nuclear translation: what is the evidence? RNA 9:1-8

David A, Dolan BP, Hickman HD, Knowlton JJ, Clavarino G, Pierre P, Bennink JR, Yewdell JW (2012) Nuclear translation visualized by ribosome-bound nascent chain puromycylation. J Cell Biol 197:45-57

Dolan BP, Knowlton JJ, David A, Bennink JR, Yewdell JW (2010a) RNA polymerase II inhibitors dissociate antigenic peptide generation from normal viral protein synthesis: a role for nuclear translation in defective ribosomal product synthesis? J Immunol 185:6728-6733

Dolan BP, Li L, Takeda K, Bennink JR, Yewdell JW (2010b) Defective ribosomal products are the major source of antigenic peptides endogenously generated from influenza A virus neuraminidase. J Immunol 184:1419-1424

Dolan BP, Sharma AA, Gibbs JS, Cunningham TJ, Bennink JR, Yewdell JW (2012) MHC class I antigen processing distinguishes endogenous antigens based on their translation from cellular versus viral mRNA. Proc Natl Acad Sci USA 109:7025-7030

Elliott T, Bodmer H, Townsend A (1996) Recognition of out-of-frame major histocompatibility complex class I-restricted epitopes in vivo. Eur J Immunol 26:1175-1179

Esquivel F, Yewdell JW, Bennink JR (1992) RMA/S cells present endogenously synthesized cytosolic proteins to class I-restricted cytotoxic T lymphocytes. J Exp Med 175:163-168

Fetten JV, Roy N, Gilboa E (1991) A frameshift mutation at the NH2 terminus of the nucleoprotein gene does not affect generation of cytotoxic T lymphocyte epitopes. J Immunol 147:2697-2705

Genuth NR, Barna M (2018) The discovery of ribosome heterogeneity and its implications for gene regulation and organismal life. Mol Cell 71:364-374

Hanada K, Yewdell JW, Yang JC (2004) Immune recognition of a human renal cancer antigen through post-translational protein splicing. Nature 427:252-256

Hickman HD, Mays JW, Gibbs J, Kosik I, Magadan JG, Takeda K, Das S, Reynoso GV, Ngudiankama BF, Wei J, Shannon JP, McManus D, Yewdell JW (2018) Influenza A virus negative strand RNA is translated for CD8(+) T cell immunosurveillance. J Immunol. https://doi.org/10.4049/jimmunol.1800586

Iborra FJ, Jackson DA, Cook PR (2004) The case for nuclear translation 1. J Cell Sci 117:5713-5720

Ingolia NT, Lareau LF, Weissman JS (2011) Ribosome profiling of mouse embryonic stem cells reveals the complexity and dynamics of mammalian proteomes. Cell 147:789-802

Ingolia NT, Brar GA, Stern-Ginossar N, Harris MS, Talhouarne GJ, Jackson SE, Wills MR, Weissman JS (2014) Ribosome profiling reveals pervasive translation outside of annotated protein-coding genes. Cell Rep 8:1365-1379

Jenkins MR, Webby R, Doherty PC, Turner SJ (2006) Addition of a prominent epitope affects influenza A virus-specific $\mathrm{CD}^{+} \mathrm{T}$ cell immunodominance hierarchies when antigen is limiting. J Immunol 177:2917-2925
Karttunen J, Sanderson S, Shastri N (1992) Detection of rare antigenpresenting cells by the lacZ T-cell activation assay suggests an expression cloning strategy for T-cell antigens. Proc Natl Acad Sci USA 89:6020-6024

Kim TS, Sun J, Braciale TJ (2011) T cell responses during influenza infection: getting and keeping control. Trends Immunol $32: 225-231$

Lev A, Princiotta MF, Zanker D, Takeda K, Gibbs JS, Kumagai C, Waffarn E, Dolan BP, Burgevin A, Van Endert P, Chen W, Bennink JR, Yewdell JW (2010) Compartmentalized MHC class I antigen processing enhances immunosurveillance by circumventing the law of mass action. Proc Natl Acad Sci USA 107:6964-6969

Liepe J, Marino F, Sidney J, Jeko A, Bunting DE, Sette A, Kloetzel PM, Stumpf MPH, Heck AJR, Mishto M (2016) A large fraction of HLA class I ligands are proteasome-generated spliced peptides. Science 354:354-358

McAllister AK (2014) Major histocompatibility complex I in brain development and schizophrenia. Biol Psychiatry 75:262-268

McGlincy NJ, Ingolia NT (2017) Transcriptome-wide measurement of translation by ribosome profiling. Methods 126:112-129

McMichael AJ, Gotch FM, Noble GR, Beare PA (1983) Cytotoxic T-cell immunity to influenza. N Engl J Med 309:13-17

Porgador A, Yewdell JW, Deng Y, Bennink JR, Germain RN (1997) Localization, quantitation, and in situ detection of specific peptide-MHC class I complexes using a monoclonal antibody. Immunity 6:715-726

Princiotta MF, Finzi D, Qian SB, Gibbs J, Schuchmann S, Buttgereit F, Bennink JR, Yewdell JW (2003) Quantitating protein synthesis, degradation, and endogenous antigen processing. Immunity 18:343-354

Reid DW, Nicchitta CV (2012) The enduring enigma of nuclear translation. J Cell Biol 197:7-9

Schubert U, Anton LC, Gibbs J, Norbury CC, Yewdell JW, Bennink JR (2000) Rapid degradation of a large fraction of newly synthesized proteins by proteasomes. Nature 404:770-774

Schuren AB, Costa AI, Wiertz EJ (2016) Recent advances in viral evasion of the MHC Class I processing pathway. Curr Opin Immunol 40:43-50

Schwanhäusser B, Busse D, Li N, Dittmar G, Schuchhardt J, Wolf J, Chen W, Selbach M (2011) Global quantification of mammalian gene expression control. Nature 473:337-342

Shi Z, Fujii K, Kovary KM, Genuth NR, Rost HL, Teruel MN, Barna M (2017) Heterogeneous ribosomes preferentially translate distinct subpools of mRNAs genome-wide. Mol Cell 67(71-83):e77

Slavov N, Semrau S, Airoldi E, Budnik B, van Oudenaarden A (2015) Differential stoichiometry among core ribosomal proteins. Cell Rep 13:865-873

Souquette A, Thomas PG (2018) Past life and future effects-how heterologous infections alter immunity to influenza viruses. Front Immunol 9:1071

Starck SR, Jiang V, Pavon-Eternod M, Prasad S, McCarthy B, Pan T, Shastri N (2012) Leucine-tRNA initiates at CUG start codons for protein synthesis and presentation by MHC class I. Science 336:1719-1723

Starck SR, Tsai JC, Chen K, Shodiya M, Wang L, Yahiro K, MartinsGreen M, Shastri N, Walter P (2016) Translation from the 5' untranslated region shapes the integrated stress response. Science 351:aad3867

Townsend ARM, Gotch FM, Davey J (1985) Cytotoxic T cells recognize fragments of the influenza nucleoprotein. Cell 42:457-467

Townsend ARM, Bastin J, Gould K, Brownlee GG (1986) Cytotoxic $\mathrm{T}$ lymphocytes recognize influenza hemagglutinin that lacks a signal sequence. Nature 324:575-577 
Vigneron N, Stroobant V, Chapiro J, Ooms A, Degiovanni G, Morel S, van der Bruggen P, Boon T, Van den Eynde BJ (2004) An antigenic peptide produced by peptide splicing in the proteasome. Science 304:587-590

Wang F, Durfee Larissa A, Huibregtse Jon M (2013) A cotranslational ubiquitination pathway for quality control of misfolded proteins. Mol Cell 50:378-389

Warren EH, Vigneron NJ, Gavin MA, Coulie PG, Stroobant V, Dalet A, Tykodi SS, Xuereb SM, Mito JK, Riddell SR, Van den Eynde BJ (2006) An antigen produced by splicing of noncontiguous peptides in the reverse order. Science 313:1444-1447

Webby RJ, Andreansky S, Stambas J, Rehg JE, Webster RG, Doherty PC, Turner SJ (2003) Protection and compensation in the influenza virus-specific $\mathrm{CD}^{+} \mathrm{T}$ cell response. Proc Natl Acad Sci USA 100:7235-7240

Wei J, Yewdell JW (2018) Immunoribosomes: Where's there's fire, there's fire. Mol Immunol. https://doi.org/10.1016/j.molimm. 2017.12.026

Wheatley DN, Giddings MR, Inglis MS (1980) Kinetics of degradation of "short-" and "long-lived" proteins in cultured mammalian cells. Cell Biol Int Rep 4:1081-1090

$\mathrm{Wu} \mathrm{T}$ (2017) Antigen presentation during influenza virus infection. Dissertation, Monash University

Yang N, Gibbs JS, Hickman HD, Reynoso GV, Ghosh AK, Bennink JR, Yewdell JW (2016) Defining viral defective ribosomal products: standard and alternative translation initiation events generate a common peptide from Influenza A virus M2 and M1 mRNAs. J Immunol 196:3608-3617
Yewdell JW (2001) Not such a dismal science: the economics of protein synthesis, folding, degradation and antigen processing. Trends Cell Biol 11:294-297

Yewdell JW (2003) Immunology. Hide and seek in the peptidome. Science 301:1334-1335

Yewdell JW (2011) DRiPs solidify: progress in understanding endogenous MHC class I antigen processing. Trends Immunol 32:548-558

Yewdell JW, Nicchitta CV (2006) The DRiP hypothesis decennial: support, controversy, refinement and extension. Trends Immunol $27: 368-373$

Yewdell JW, Anton LC, Bennink JR (1996) Defective ribosomal products (DRiPs): a major source of antigenic peptides for MHC class I molecules? J Immunol 157:1823-1826

Zanker D, Waithman J, Yewdell JW, Chen W (2013) Mixed proteasomes function to increase viral peptide diversity and broaden antiviral $\mathrm{CD}^{+}{ }^{+} \mathrm{T}$ cell responses. J Immunol 191:52-59

Zhirnov OP, Poyarkov SV, Vorob'eva IV, Safonova OA, Malyshev NA, Klenk HD (2007) Segment NS of influenza a virus contains an additional gene NSP in positive-sense orientation. Doklady Biochem Biophys 414:127-133

Zinkernagel RM, Doherty PC (1974) Restriction of in vitro T cellmediated cytotoxicity in lymphocytic choriomeningitis within a syngeneic or semiallogeneic system. Nature 248:701-702

Zook MB, Howard MT, Sinnathamby G, Atkins JF, Eisenlohr LC (2006) Epitopes derived by incidental translational frame shifting give rise to a protective CTL response. J Immunol 176:6928-6934 